\title{
Search for the 531-day-period wobble signal in the polar motion based on EEMD
}

\author{
H. Ding ${ }^{1}$ and W. Shen ${ }^{1,2}$ \\ ${ }^{1}$ School of Geodesy and Geomatics, Key Laboratory of Geospace Environment and Geodesy of the Ministry of Education, \\ Wuhan University, Wuhan 430079, China \\ ${ }^{2}$ State Key Laboratory of Information Engineering in Surveying, Mapping and Remote Sensing, China
}

Correspondence to: W. Shen (wbshen@sgg.whu.edu.cn)

Received: 22 March 2015 - Published in Nonlin. Processes Geophys. Discuss.: 29 April 2015

Revised: 15 July 2015 - Accepted: 18 July 2015 - Published: 5 August 2015

\begin{abstract}
In this study, we use a nonlinear and nonstationary time series analysis method, the ensemble empirical mode decomposition method (EEMD), to analyze the polar motion (PM) time series (EOP C04 series from 1962 to 2013) to find a 531-day-period wobble $(531 \mathrm{dW})$ signal. The $531 \mathrm{dW}$ signal has been found in the early PM series (1962-1977), but cannot be found in the recent PM series (1978-2013) using conventional analysis approaches. By virtue of the demodulation feature of EEMD, the $531 \mathrm{dW}$ can be confirmed to be present in PM based on the differences of the amplitudes and phases between different intrinsic mode functions. Results from three sub-series divided from the EOP C04 series show that the period of the $531 \mathrm{dW}$ is subject to variations, in the range of 530.9-524 days, and its amplitude is also time-dependent (about 2-11 mas). Synthetic tests are carried out to explain why the $531 \mathrm{dW}$ can only be observed in recent 30-year PM time series after using EEMD. The $531 \mathrm{dW}$ is also detected in the two longest available superconducting gravimeter (SG) records, which further confirms the presence of the $531 \mathrm{dW}$. The confirmation of the $531 \mathrm{dW}$ existence could be significant in establishing a more reasonable Earth rotation model and may effectively contribute to the prediction of the PM and its mechanism interpretation.
\end{abstract}

\section{Introduction}

It is recognized that the polar motion (PM) contains two dominant components: the annual wobble (AW) with a 12month period and the Chandler wobble $(\mathrm{CW})$ with a 14 month period. Some researchers have suggested that the $\mathrm{CW}$ is highly variable with respect to its amplitude (e.g., Carter, 1981, 1982; Höpfner, 2003; Chen et al., 2009, 2013a, b), some have considered it to have double or multiple frequencies (e.g., Chao, 1983; Pan, 2012), and some have considered its frequency to be invariant (e.g., Okubo, 1982; Vicente and Wilson, 1997; Gross et al., 2003; Seitz and Schmidt, 2005). If the CW is frequency modulated as Carter (1981, 1982) suggested, namely, the frequency is governed by the magnitude, it will create an infinite number of sidebands, arranged symmetrically about the carrier and spaced at integer multiples of the modulating frequency (Carter, 1981). The first upper and lower sidebands could be at $1 \mathrm{cpy}$ (cycles per year) and $0.69 \mathrm{cpy}$, respectively, when the beat frequency is 0.157 cpy (CW's frequency $0.843 \mathrm{cpy} \pm k \times 0.157$; $k=1$ denotes the first upper and lower sidebands), where the beat frequency or beat period is the time period required for the Earth's pole to complete a cycle of the combined AW (12 months) and CW (14 months). Because the first upper sideband of the $\mathrm{CW}$ was contaminated in the spectrum of the AW, it was concluded that only the first lower sideband, which is located at $0.686 \mathrm{cpy}$, could most likely be detected. Based on a 16-year time series of International Polar Motion Data (spanning from 1962 to 1977 ), a 0.686 cpy component with its amplitude being around 10 to 17 mas (milliarcsecond) was very weakly detected in Carter (1982), but its signal-to-noise ratio (SNR) is very low. Based on the 
PM series (spanning from 1974 to 1981) obtained by the Doppler satellite tracking and lunar laser ranging, Morgan et al. (1982) identified two spectral peaks at $532 \pm 10.8$ days and $537 \pm 15.2$ days, with their amplitudes around $8.6 \pm 2.0$ and $7.4 \pm 2.2 \mathrm{mas}$, respectively. However, after then (1980s), this signal was seldom announced in the PM time series with higher SNR (especially for the records after the mid-1990s). Recently, Na et al. (2011) found a 500-day period component in the PM data with an average amplitude of 20 mas, and argued that, if assuming the existence of this component, the RMS error of the prediction of the PM could be reduced by $50 \%$, and suggested that this phenomenon should be caused by resonance of an unidentified oscillating mode of the Earth (possibly the Earth's inner core wobble). In addition, this wobble (or referred to as an 18-month wobble) was found in the analysis of the atmospheric angular momentum data by Wahr (1983) and Chen et al. (2010). Furthermore, a signal with a period of about 1.5 years could also be found from the variation of length of day (LOD) by using the wavelet analysis as suggested by Chao et al. (2014). To check our results (this paper), King (2015) used a Kalman filter smoother to analyze the PM series in the time domain, and his results show that the amplitude and especially the phase of the $531 \mathrm{dW}$ are time-variable (www. nonlin-processes-geophys-discuss.net/2/C163/2015/), while relevant earlier results using a Kalman filter on the PM series can be found in King and Watson (2014). Besides these, to our knowledge, this component was poorly studied. Inasmuch as the detection of this component could be significant in establishing a more reasonable Earth rotation model and may effectively contribute to the prediction of the PM, the main purpose of this study is to detect the $\sim 530$-day-period wobble (simply $531 \mathrm{dW}$ hereafter for convenience, the reason for which can be found in Sect. 3) component and to provide a possible explanation why in general cases it could not be detected from observation series (e.g., EOP C04 series) based on the Fourier analysis.

Generally, the traditional Fourier analysis method cannot observe this $531 \mathrm{dW}$ signal (see Fig. 2d-f); hence, in this study, a nonlinear and non-stationary time series analysis method, the ensemble empirical mode decomposition (EEMD) (Huang and Wu, 2008; Wu and Huang, 2009), is applied to a PM time series as a filter bank to detect the $531 \mathrm{dW}$ signal. (To know the differences and relations between different spectral analysis methods, we suggest the readers to refer some other literatures, such as Tary et al., 2014).

Here, the PM time series, the EOP C04 series spanning from 1962 to 2013 with a 1-day sampling interval from the International Earth Rotation and Reference System Service (IERS) (http://www.iers.org/IERS/EN/ DataProducts/EarthOrientationData/), is used for observing the signal of interest.

\section{Method}

EEMD was proposed to overcome the disadvantages existing in the empirical mode decomposition (EMD) (Huang et al., 1998; Huang and Wu, 2008), such as the mode-mixing problem and the end effect (see Fig. 2 in Shen and Ding (2014)), although EMD has demonstrated its applicability in a wide range of geoscience studies over the last 15 years (e.g., Pee and McMahon, 2006; Thomas et al., 2009; Franzke, 2009; Jackson and Mound, 2010; Jeng and Chen, 2011; Lee et al., 2011; Shen and Ding, 2014; Chambers, 2015). The details of EMD and EEMD can be found in many relevant literatures (e.g., Huang et al., 1998; Huang and Wu, 2008; Wu and Huang, 2009; Shen and Ding, 2014).

A given time series $x(t)$ can be decomposed into a number of intrinsic mode functions (IMFs) using the following steps (Huang and Wu, 2008; Shen and Ding, 2014).

1. Identify all local maxima and local minima of $x(t)$, where its upper (lower) envelope can be formed using a cubic spline line to connect all the local maxima (minima).

2. Use $h_{1}(t)=x(t)-m_{1}(t)$ to get a new series, where $m_{1}(t)$ is the mean of the upper and lower envelopes of $x(t)$. Steps 1 and 2 are called a sifting procedure.

3. Generally, $h_{1}(t)$ is not an IMF; hence, treat $h_{1}(t)$ as the data given just as $x(t)$, and repeat the sifting procedure (steps 1 and 2) $k$ times until $h_{k}(t)$ is an IMF, namely, $h_{1 k}(t)=h_{1(k-1)}(t)-m_{1 k}(t)$. Let $c_{1}(t)=h_{1 k}(t)$, which is designated as the first IMF.

4. Then, letting $r_{1}(t)=x(t)-c_{1}(t)$, and repeating steps 1-3 for $r_{1}(t)$, one can get the second IMF, $c_{2}(t)$.

5. Repeat the above steps, and then the $j$ th target IMF, $c_{j}(t)$, can be obtained.

The above process is the EMD. In these steps, two different iterative loops exist. The first loop is employed to obtain $h_{k}(t)$, in which an IMF must have a specific definition to stop circulating steps 1 and 2 . In the second loop, the repeat times $j$ must be a finite number, in which a criterion must be determined to stop the entire sifting process. For the first loop, Huang et al. (1998) limited the size of the standard deviation, $\mathrm{SD}_{k}=\sum_{t=0}^{T}\left|m_{1 k}\right|^{2} / \sum_{t=0}^{T}\left|h_{1 k}\right|^{2}$, which was computed from the two consecutive sifting results to stop this loop. $\mathrm{SD}_{k}$ can generally be set between 0.2 and 0.3 . The second loop can be terminated by either of the two criteria: (1) the component $c_{n}$ or the residue $r_{n}$ becomes so small that it is less than the previously given value of the substantial consequence; or (2) no more IMF can be extracted from $r_{n}$ (see also Shen and Ding, 2014).

Given that EMD has the mode-mixing problem and end effects, EEMD is developed with the following procedures (Huang and Wu, 2008; Shen and Ding, 2014). 
1. Add a white noise series to the targeted time series $x(t)$.

2. Decompose the series with added white noise into IMFs.

3. Repeat procedures 1 and 2 iteratively, but with different white noise series each time.

4. Obtain the (ensemble) means of the corresponding IMFs of the decompositions as the final IMF.

In the decomposition using EEMD, the added white noise series cancel each other out in the final mean of the corresponding IMF. Note that in the EEMD process, procedure 2 is just the whole EMD process. The means of the IMFs remain within the natural filter windows, significantly reducing the possibility of mode mixing and preserving the filter property (Huang and Wu, 2008; Shen and Ding, 2014).

By using EEMD, the chosen time series can be decomposed into a finite number of simple IMFs, and differentscale signals in the series will be re-combined by proper IMFs based on the fact that different IMFs have different frequency bands. Hence, for the chosen EOP C04 series, $f(t)$ and its IMFs satisfy the following linear equation:

$f(t)=\operatorname{IMF} 1(t)+\operatorname{IMF} 2(t)+\ldots+\operatorname{IMF} n(t)+r(t)$,

where $n$ is the number of the IMFs and $r(t)$ is a residue term. In addition, EEMD can be used to demodulate a frequencymodulated time series (Huang et al., 1998; Huang and Wu, 2008). That means EEMD can be used not only as a filter bank, but also as a demodulator simultaneously. Since EEMD has these two advantages, we use it to detect the $531 \mathrm{dW}$ in the PM.

A frequency modulation signal can be expressed as follows (Carter, 1981, 1982):

$e_{t}(x, y)=C_{\mathrm{c}} \sin \left[\phi_{0}+2 \pi f_{\mathrm{c}} t+M \cdot \sin \left(2 \pi f_{\mathrm{m}} t\right)\right]$,

where $e_{t}(x, y)$ is the expected value of the $x$-component or $y$ component, and $f_{\mathrm{m}}$ the frequency of the modulating signal. Here, we set it to be equal to $0.157 \mathrm{cpy}$ (the beat frequency between the $\mathrm{CW}$ and $\mathrm{AW}) ; f_{\mathrm{c}}$ and $C_{\mathrm{c}}$ are the frequency and amplitude of the $\mathrm{CW}$, and $M$ is the modulation index, defined as $M=\Delta f / f_{\mathrm{m}}$, where $\Delta f$ is the maximum variation of $f_{\mathrm{m}} ; \phi_{0}$ is the initial phase, and it is simply set as zero (same as the AW and the $531 \mathrm{dW}$ ).

\section{Results}

\subsection{Results from the PM series with/without using EEMD}

The waveforms of the two components, the $x$ - and $y$ components of the chosen PM series are shown in Fig. 1a, and their corresponding Fourier spectra are shown in Fig. 1b, c (see the black bold curves; we call them the original spectra). The vertical dashed lines (Fig. 1b and c) are located at the target frequency, $0.6875 \mathrm{cpy}$, and there is no peak that can be identified for the $531 \mathrm{dW}$ based on the original spectra of PM series. Meanwhile, if the EEMD is used on these two components, 11 IMFs can be obtained, respectively (see Fig. 2 for the waveforms of the IMFs of the $x$-component). In the Fourier spectra of IMF5 and IMF6, the $531 \mathrm{dW}$ can be clearly detected (see the blue and red curves in Fig. $1 \mathrm{~b}$ and c). The spectra for other IMFs (sum) of the $x$ - and $y$-components are denoted by the green curves (we call them the residual spectra). No peak can be identified for the $531 \mathrm{dW}$ in the residual spectra. The amplitudes of the $531 \mathrm{dW}$ signals in IMF5 and IMF6 are denoted by the blue and red arrows, respectively. Comparing with the original spectra, the amplitudes in IMF5 and IMF6 are outstanding (Fig. 1b and c); the phases of the IMF5 and IMF6 are almost opposite (Fig. 1d and e; it might be caused by the demodulation feature of EEMD). According to Eq. (1), the mean amplitudes of $531 \mathrm{dW}$ in the $x$ - and $y$-components achieve 7.1 and 7.2 mas, respectively, while the corresponding noise level is about 4 mas. The low SNR might be the reason why we cannot identify the $531 \mathrm{dW}$ signal directly from the original spectra of PM time series spanning from 1962 to 2013 using conventional analysis approaches. However, previous studies show that this signal has been found in the 1962-1977 PM series (see Carter, 1981, 1982). One possible inference is that the amplitude of the $531 \mathrm{dW}$ may vary with time. Hence, we divide the 1962-2013 PM series into three sub-series, the 1962-1977 series, the 1978-1994 series, and the 19952013 series, to further study the $531 \mathrm{dW}$ signal. Note that, after 1980, the EOP time series is generated from the modern geodetic observations, and the precision of the time series has been highly improved. However, given that the EEMD is actually based on the waveform of the time series to filter different signals for different IMFs, we have confirmed that the distinction at 1980 will not significantly affect our results.

Based on the conventional Fourier analysis approach, the results as shown in Fig. 3 clearly indicate that only the target peak in the spectra of the 1962-1977 series (see Fig. 3b) is over their corresponding background noise level (see Fig. 3d; note that a 0.57 cpy peak in Fig. $3 \mathrm{f}$ is also over the noise level, but it is not the interesting signal of this study), which is consistent with previous studies (Carter, 1981, 1982; Morgan et al., 1982). Without using EEMD, our estimates for the target $531 \mathrm{dW}, \mathrm{CW}$, and AW (the annual wobble) are tabulated in Table 1 (note that the estimations for the amplitudes, frequencies and their corresponding error bars are obtained by using the AR (Autoregressive) method of Chao and Gilbert, 1980; more details can be found in Ding and Shen, 2013, and Ding and Chao, 2015). For the $x$ - and $y$-components of the $531 \mathrm{dW}$ signal from the 1962-1977 series, the corresponding amplitudes are 11.3 and 14.6 mas, while the estimates of previous studies are about 8 mas (Carter, 1981, 1982; Morgan 

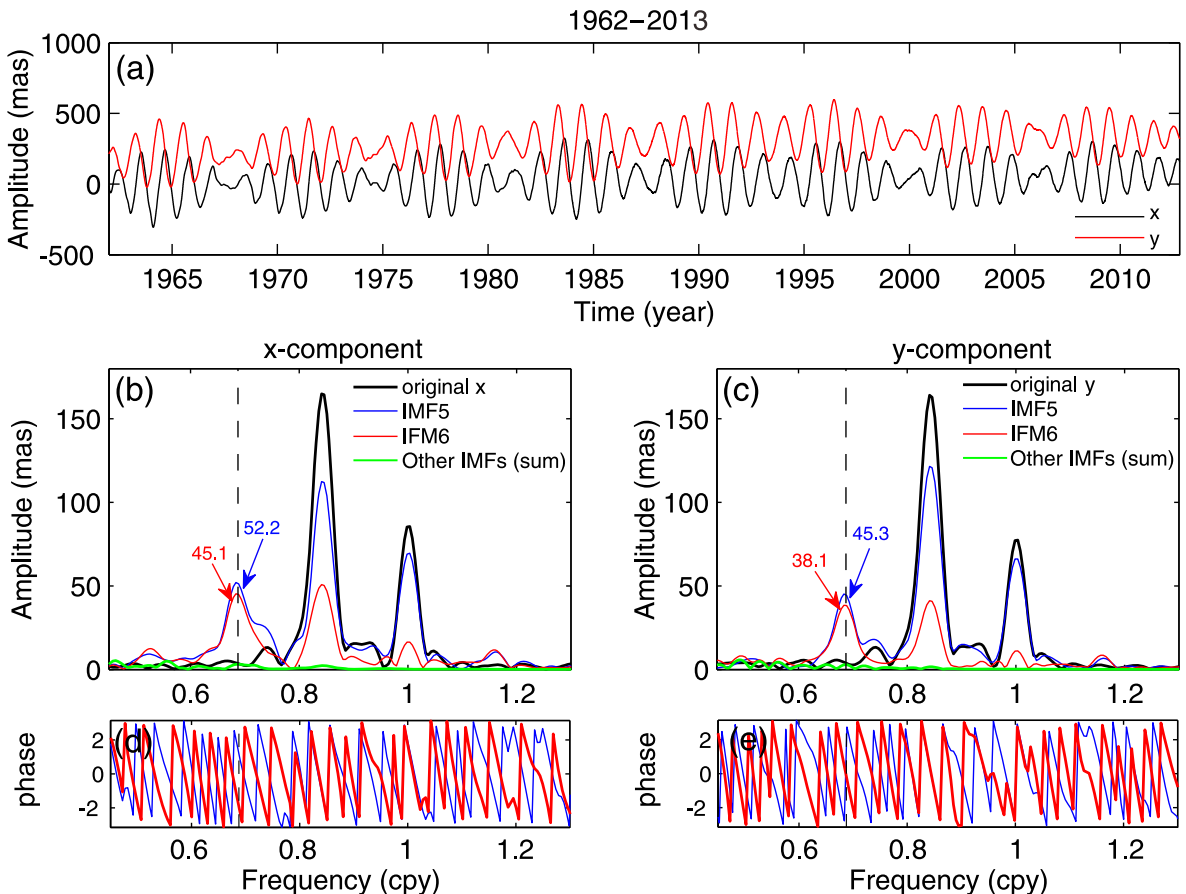

Figure 1. The $x$ - (black) and $y$-components (red) of the 1962-2013 PM series (a) and their corresponding Fourier amplitude spectra (original spectra, denoted by the black curves in $\mathbf{b}$ and c). (b) $x$-component; (c) $y$-component. The spectra for IMF5 and IMF6 after applying EEMD to $x$ - and $y$-components are indicated by blue and red curves, and their corresponding phases (based on the discrete Fourier transform) are shown in (d) and (e), respectively. The spectra (residual spectra) for other IMFs (sum) of $x$ - and $y$-components are denoted by the green curves.
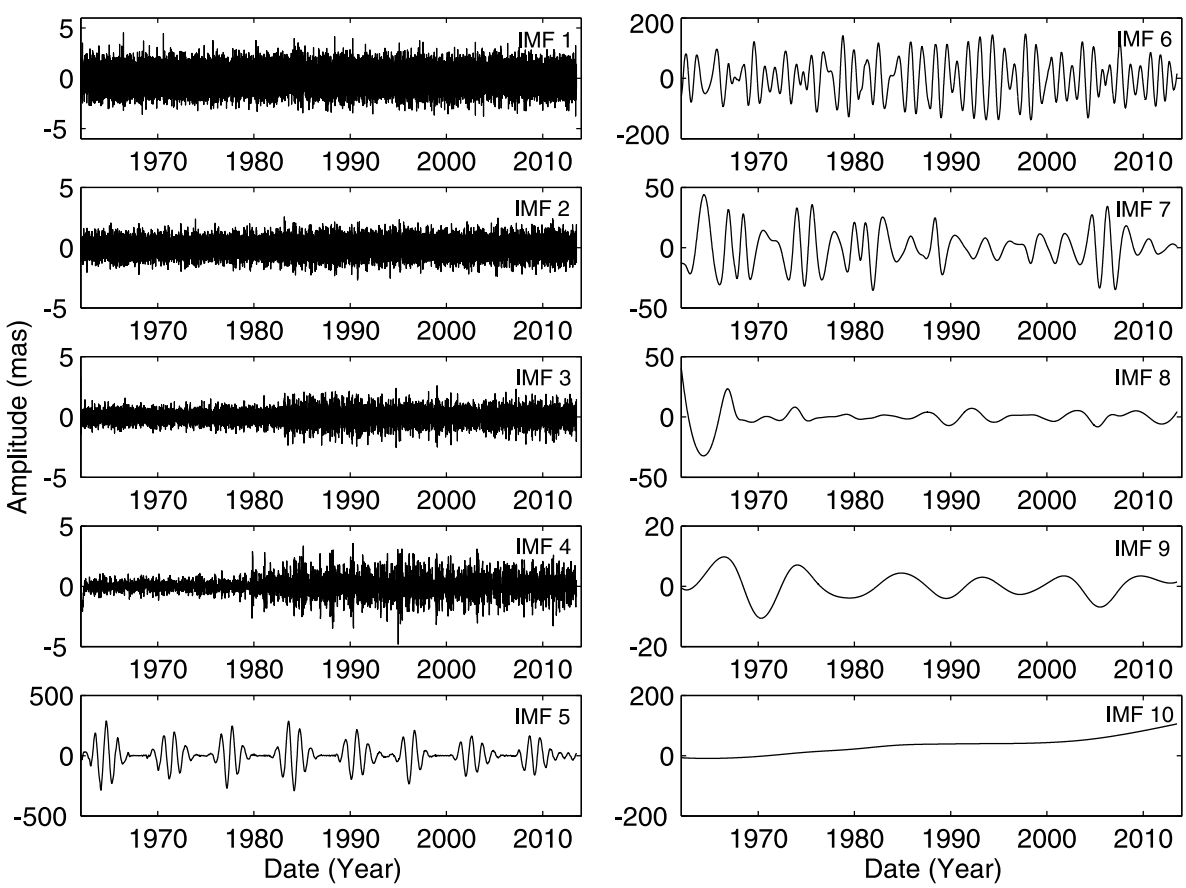

Figure 2. The first 10 IMFs of the $x$-component of the 1962-2013 PM time series after using EEMD; the last one, IMF11, is a linear term. Here, it is ignored. 
Table 1. The observed frequencies (cpy) and amplitudes (mas) of the CW, AW and the target wobble.

\begin{tabular}{|c|c|c|c|c|c|c|c|}
\hline & \multicolumn{2}{|c|}{ Target wobble } & \multicolumn{2}{|c|}{ Chandler wobble } & \multicolumn{2}{|c|}{ Annual wobble } \\
\hline & & Frequency & Amplitude & Frequency & Amplitude & Frequency & Amplitude \\
\hline \multirow[t]{6}{*}{$1962-1977$} & $x$-component* & $0.68751 \pm 3.2 \times 10^{-4}$ & $11.3 \pm 4.6$ & $0.84381 \pm 2.4 \times 10^{-4}$ & $129.2 \pm 3.3$ & $1.00023 \pm 2.6 \times 10^{-4}$ & $97.1 \pm 4.1$ \\
\hline & $x$-IMF5 & $0.68749 \pm 3.4 \times 10^{-4}$ & $44.1 \pm 5.1$ & $0.84380 \pm 2.6 \times 10^{-4}$ & $103.6 \pm 3.4$ & $1.00019 \pm 3.1 \times 10^{-4}$ & $73.1 \pm 4.5$ \\
\hline & $x$-IMF6 & $0.68750 \pm 4.7 \times 10^{-4}$ & $33.2 \pm 7.5$ & $0.84381 \pm 9.8 \times 10^{-4}$ & $25.1 \pm 7.9$ & $1.00021 \pm 9.9 \times 10^{-4}$ & $24.6 \pm 8.3$ \\
\hline & $y$-component* & $0.68753 \pm 3.4 \times 10^{-4}$ & $14.6 \pm 4.8$ & $0.84383 \pm 2.7 \times 10^{-4}$ & $129.2 \pm 3.2$ & $1.00028 \pm 3.1 \times 10^{-4}$ & $90.8 \pm 3.9$ \\
\hline & $y$-IMF5 & $0.68752 \pm 3.6 \times 10^{-4}$ & $44.9 \pm 5.3$ & $0.84384 \pm 2.8 \times 10^{-4}$ & $104.0 \pm 3.3$ & $1.00027 \pm 3.3 \times 10^{-4}$ & $73.2 \pm 4.7$ \\
\hline & $y$-IMF6 & $0.68753 \pm 4.5 \times 10^{-4}$ & $32.7 \pm 7.3$ & $0.84384 \pm 1.0 \times 10^{-3}$ & $23.8 \pm 8.2$ & $1.00031 \pm 1.2 \times 10^{-3}$ & $18.5 \pm 9.0$ \\
\hline \multirow[t]{6}{*}{ 1978-1994 } & $x$-component* & - & - & $0.84312 \pm 1.7 \times 10^{-4}$ & $180.1 \pm 2.1$ & $1.00031 \pm 2.4 \times 10^{-4}$ & $90.6 \pm 3.4$ \\
\hline & $x$-IMF5 & $0.69614 \pm 3.5 \times 10^{-4}$ & $50.0 \pm 4.0$ & $0.84311 \pm 2.1 \times 10^{-4}$ & $145.5 \pm 2.6$ & $1.00027 \pm 3.0 \times 10^{-4}$ & $83.9 \pm 3.5$ \\
\hline & $x$-IMF6 & $0.69611 \pm 3.7 \times 10^{-4}$ & $45.3 \pm 5.3$ & $0.84309 \pm 7.2 \times 10^{-4}$ & $27.2 \pm 6.7$ & - & - \\
\hline & $y$-component* & - & - & $0.84314 \pm 1.8 \times 10^{-4}$ & $180.1 \pm 2.2$ & $1.00029 \pm 2.7 \times 10^{-4}$ & $84.1 \pm 3.5$ \\
\hline & $y$-IMF5 & $0.69617 \pm 3.7 \times 10^{-4}$ & $50.5 \pm 3.9$ & $0.84316 \pm 2.0 \times 10^{-4}$ & $153.3 \pm 2.5$ & $1.00028 \pm 3.2 \times 10^{-4}$ & $82.0 \pm 3.7$ \\
\hline & $y$-IMF6 & $0.69613 \pm 3.9 \times 10^{-4}$ & $44.1 \pm 4.2$ & $0.84314 \pm 6.4 \times 10^{-4}$ & $29.9 \pm 5.9$ & - & - \\
\hline \multirow[t]{6}{*}{ 1995-2013 } & $x$-component* & - & - & $0.83892 \pm 2.5 \times 10^{-4}$ & $128.0 \pm 3.4$ & $1.00030 \pm 3.6 \times 10^{-4}$ & $100.8 \pm 4.5$ \\
\hline & $x$-IMF5 & $0.68644 \pm 4.9 \times 10^{-4}$ & $31.2 \pm 7.0$ & $0.83895 \pm 3.1 \times 10^{-4}$ & $81.1 \pm 4.5$ & $1.00025 \pm 4.3 \times 10^{-4}$ & $63.0 \pm 5.3$ \\
\hline & $x$-IMF6 & $0.68646 \pm 7.7 \times 10^{-4}$ & $33.3 \pm 6.9$ & $0.83893 \pm 6.8 \times 10^{-4}$ & $46.6 \pm 5.8$ & $1.00031 \pm 7.4 \times 10^{-4}$ & $39.2 \pm 6.7$ \\
\hline & $y$-component* & - & - & $0.83893 \pm 2.4 \times 10^{-4}$ & $128.2 \pm 3.2$ & $1.00027 \pm 3.8 \times 10^{-4}$ & $91.8 \pm 5.1$ \\
\hline & $y$-IMF5 & $0.68648 \pm 4.7 \times 10^{-4}$ & $32.8 \pm 6.5$ & $0.83897 \pm 2.9 \times 10^{-4}$ & $86.6 \pm 4.1$ & $1.00027 \pm 4.1 \times 10^{-4}$ & $63.6 \pm 5.7$ \\
\hline & $y$-IMF6 & $0.68644 \pm 6.1 \times 10^{-4}$ & $33.3 \pm 6.4$ & $0.83894 \pm 5.4 \times 10^{-4}$ & $37.9 \pm 5.5$ & $1.00031 \pm 7.4 \times 10^{-4}$ & $28.5 \pm 6.6$ \\
\hline
\end{tabular}

* Directly estimated values from observation series without using EEMD.

Table 2. The vector differences of the amplitudes of IMF 5 and IMF6 for the three sub-series (frequency domain).

\begin{tabular}{llccc}
\hline & & $1962-1977$ & $1978-1994$ & $1995-2013$ \\
\hline \multirow{2}{*}{$x$-component } & 531 dW & 12.8 & 4.0 & 3.4 \\
& RMS noise level & 9.5 & 8.8 & 5.4 \\
\hline \multirow{2}{*}{$y$-component } & 531 dW & 16.4 & 8.7 & 3.9 \\
& RMS noise level & 9.2 & 8.2 & 5.5 \\
\hline
\end{tabular}

et al., 1982). However, this wobble cannot be found in the 1978-1994 and 1995-2013 series.

After applying EEMD to the three sub-series, only IMF5 and IMF6 contain the $531 \mathrm{dW}$ signal, the corresponding spectra being shown in Fig. 4 (for the $x$-component) and Fig. 5 (for the $y$-component). As for other IMFs, the corresponding peaks at $531 \mathrm{dW}$ frequency cannot exceed the background noise levels of their spectra just as in the whole 1962-2013 PM series they cannot (see the green curves in Fig. 1). Note that we do not rule out the possibility that some part (energy) of the interesting signals may also be present in the adjacent IMFs, but they must be very weak (see Fig. 1) so that they can be neglected. Hence, in the present case, we will only concern with the IMF5 and IMF6.

As shown in Figs. 4 and 5, the phases for CW (and AW) in IMF5 and IMF6 are the same (except for IMF 6 in Figs 4c and $5 \mathrm{c}$, where there is no peak for AW), whereas the phases for the $531 \mathrm{dW}$ in IMF5 and IMF6 are quite different. The corresponding amplitude and frequency estimates are listed in Table 1. Taking into account the same phases as shown in Figs. 4 and 5, one can find that the amplitudes from the IMFs for $\mathrm{CW}$ and $\mathrm{AW}$ are consistent with the results without using EEMD (see Table 1). As for the amplitudes of the $531 \mathrm{dW}$ in the three sub-series, we use the vector difference to estimate the relevant values. The corresponding results are shown in Table 2. Considering the estimation errors, Table 2 also indicates that the $531 \mathrm{dW}$ cannot be found in the spectra by using the Fourier analysis (as shown in Fig. 3e and $\mathrm{f}$ ).

Wahr (1983) implied that there exists an 18-month $(\sim 547$ day) polar wobble (which can be considered as coinciding with the $531 \mathrm{dW}$ signal mentioned in the present paper), which could be caused by oceanic excitation. Chen et al. (2010) further demonstrated that the atmospheric excitation may give rise to the $531 \mathrm{dW}$ signal with an amplitude 15 mas using the atmospheric angular momentum (AAM) data set spanning from 1948 to 2006. But, from the above results, those excited $531 \mathrm{dW}$ signals almost disappear from the 1978-1994 and 1995-2013 series. This might be due to the fact that the $531 \mathrm{dW}$ signal has varying amplitudes and phases with time. However, after using EEMD, the $531 \mathrm{dW}$ signals can be detected in two IMFs with very different phases. Hence, we may carefully suggest that this phenomenon can be explained by the demodulation feature of EEMD. And this feature of EEMD gives us a chance to confirm that the $531 \mathrm{dW}$ is present in PM due to the differences of the phases between different IMFs. 

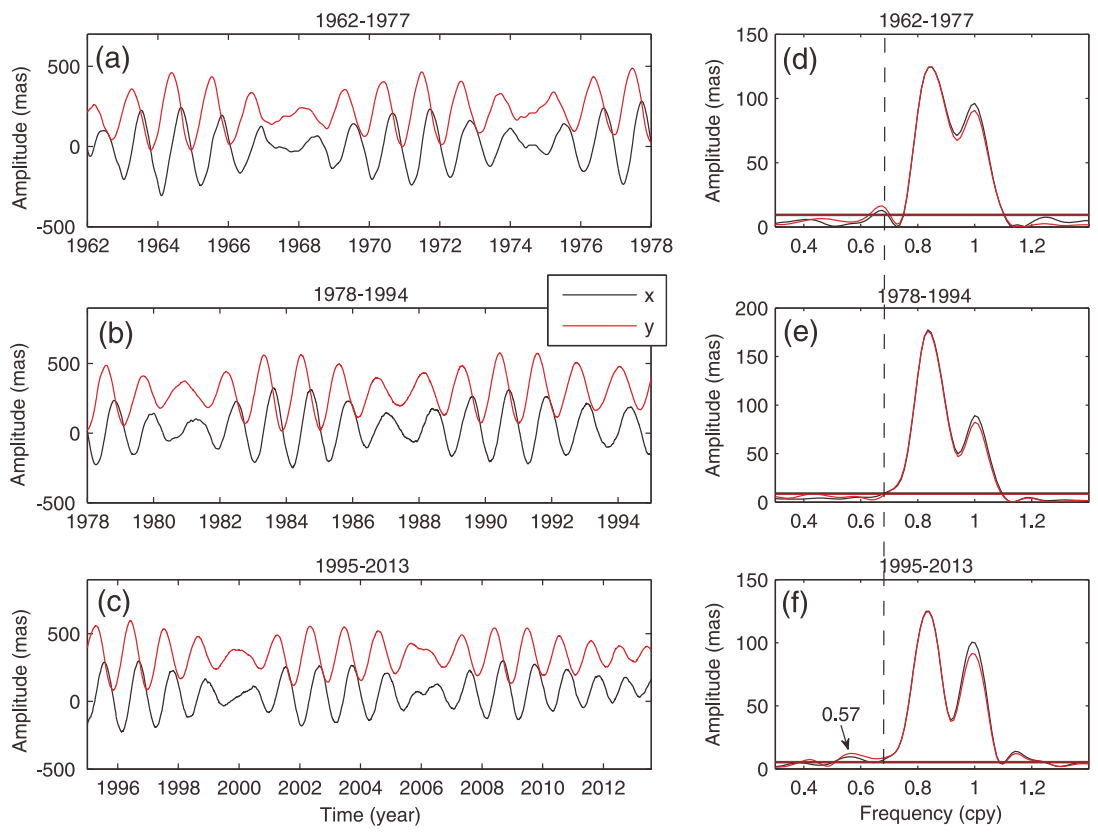

Figure 3. The three sub-series divided from the EOP C04 series (1962-2013) and their corresponding amplitude spectra. The vertical dashed line is located at $0.687 \mathrm{cpy}$, and the horizontal lines denote the RMS noise amplitudes of the frequency bands $0.2-0.75 \mathrm{cpy}$ and $1.05-2.0 \mathrm{cpy}$, which are used as the background noise levels of their corresponding spectra. For the $x$ - and $y$-components of the 1962-1977 series, the RMS noise amplitudes are 9.5 and 9.2 mas, respectively; for those of the 1978-1994 series, they are 8.8 and 8.2 mas; for those of the 1995-2013 series, they are 5.4 and 5.5 mas.
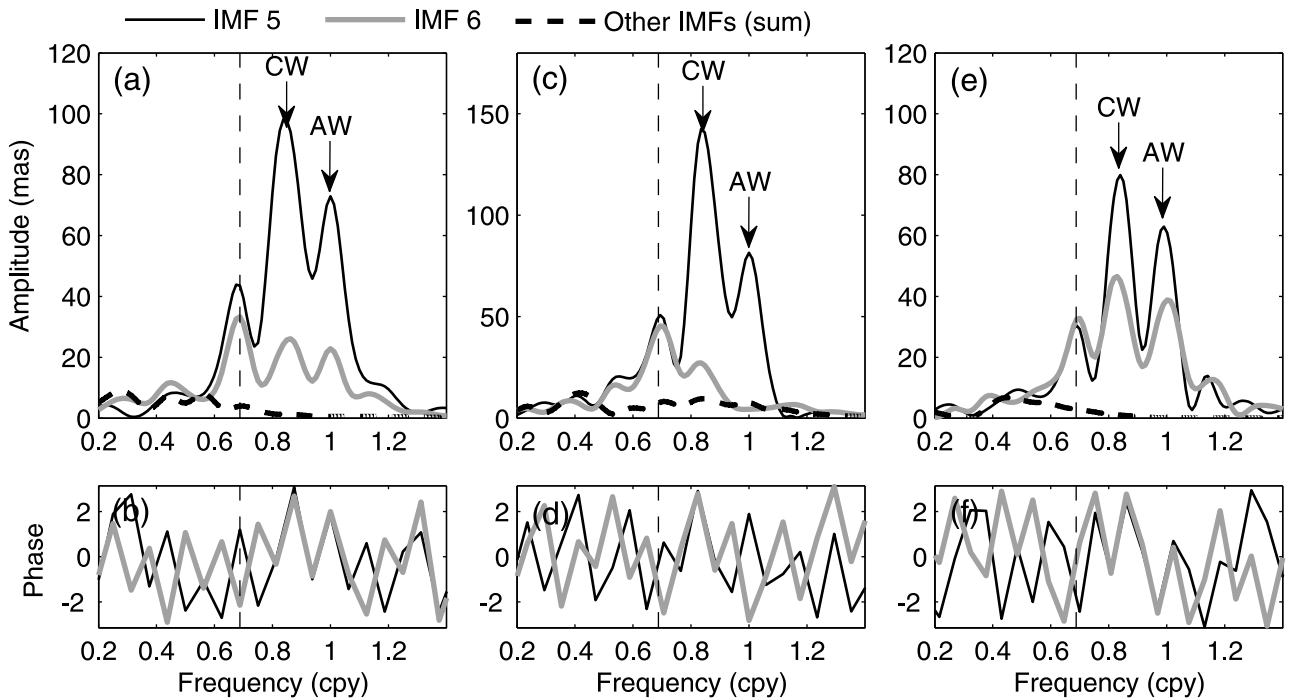

Figure 4. The amplitudes and phase spectra of the IMF 5 and IMF 6 and the sum of the other IMFs of the $x$-components of the three sub-series after using EEMD (a-f) for the 1962-1977, 1978-1994 and 1995-2013 series, respectively. The vertical dashed lines denote the possible spectral peaks for the $531 \mathrm{dW}$.

\subsection{Synthetic tests for the frequency modulation and demodulation}

By carefully examining Table 1, we can find that the amplitudes of the $531 \mathrm{dW}$ in IMF6 perhaps have some proportional relation to the amplitudes of $\mathrm{CW}$ in their corresponding $x-/ y$ - components without using EEMD (namely, those two corresponding amplitudes for $x$ - (or $y$-) component have almost linear relationship (considering the error bar); see Table 1), whereas the amplitudes of the $531 \mathrm{dW}$ in IMF5 and their corresponding $\mathrm{CW}$ amplitudes in $x$-/ $y$-components without using EEMD have no obvious linear relationship. According to 

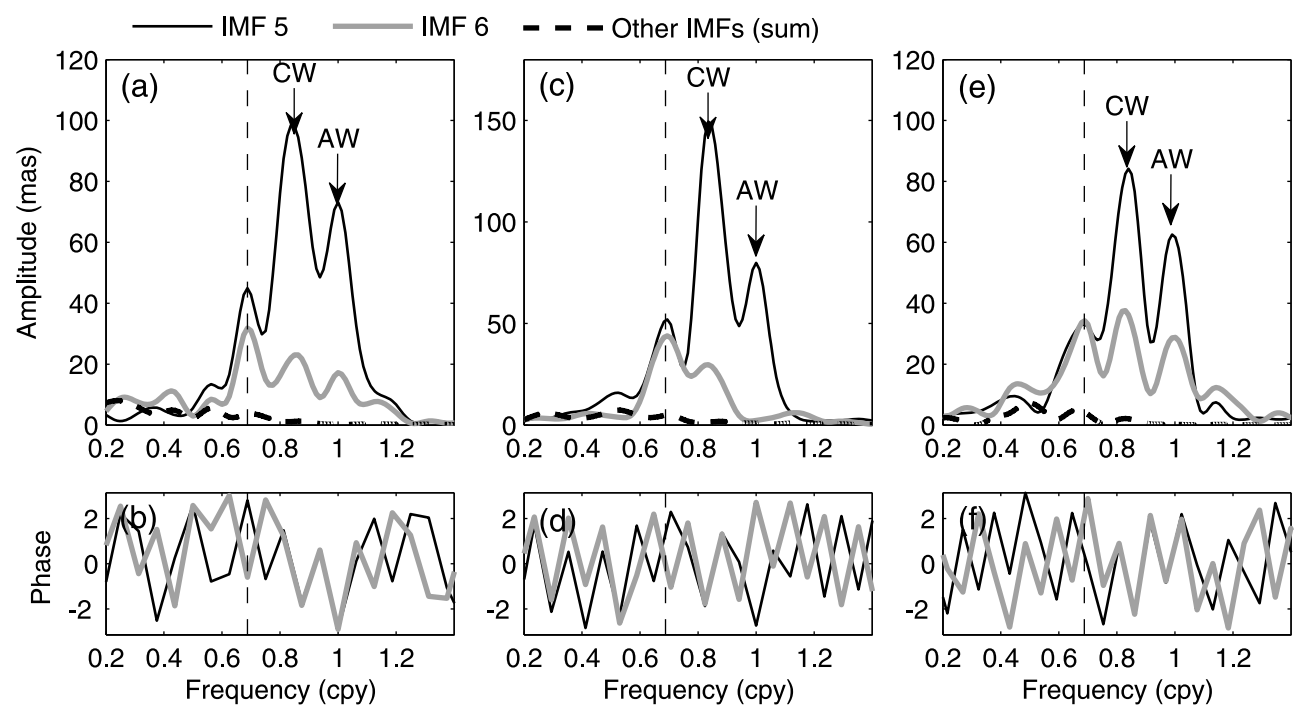

Figure 5. Same as Fig. 4 but for the $y$-components of the three sub-series.

Table 3. The chosen amplitudes of the CW (0.8437 cpy) and AW (1 cpy) for the synthetic series, and the corresponding estimated values from the synthetic series. (Unit: mas.)

\begin{tabular}{lrrrrrr}
\hline \multirow{2}{*}{$M$} & & \multicolumn{3}{c}{ Synthetic (input) } & \multicolumn{3}{c}{ Synthetic (output) } \\
\cline { 3 - 7 } & & CW & AW & CW & AW & $531 \mathrm{dW}$ \\
\hline 0.23 & $1962-1978$ & 130.9 & 82.06 & 129.2 & 97.1 & 15.17 \\
& $1978-1995$ & 181.9 & 68.25 & 180.1 & 90.6 & 19.82 \\
& $1995-2013$ & 129.5 & 85.4 & 128.0 & 100.8 & 14.89 \\
\hline 0.38 & $1962-1978$ & 133.9 & 72.03 & 129.2 & 97.1 & 25.06 \\
& $1978-1995$ & 186.3 & 54.23 & 180.1 & 90.6 & 33.49 \\
& $1995-2013$ & 132.6 & 75.6 & 128.0 & 100.8 & 24.65 \\
\hline
\end{tabular}

previous studies, here we assume that the amplitude of the $531 \mathrm{dW}$ in IMF6 is caused by the frequency modulation of CW.

The expression of the frequency modulation signal can be found in Eq. (2) in Sect. 2. From Table 1, we can get the amplitudes of the $x$ - and $y$-components of the three sub-series (without using EEMD); hence, we use those parameters to generate three synthetic noise-free time series based on Eq. (2), and they have the same sampling intervals and lengths as the 1962-1977, 1978-1994, and 1995-2013 series, respectively. As previous studies suggested (Carter, 1981, 1982), we first test the modulation index $M$ of $C W$ that equals 0.23 and 0.38 , respectively, and set different amplitudes for the $\mathrm{CW}$ and AW to ensure that the synthetic spectra coincide with the spectra of the actual observations (here we use the $x$-components of the three sub-series as examples), and the frequencies of the $\mathrm{CW}$ and $\mathrm{AW}$ are set to 0.8437 and $1.00 \mathrm{cpy}$. The set amplitude parameters (input) and their corresponding synthetic output are listed in Table 3. The corresponding spectral results are shown in Fig. 6. From Table 1 and Fig. 6 , one can clearly find that, if $M=0.23$, for the
1962-1977 series, the amplitude ( $x$-component) of the sideband that is caused by the frequency modulation of the $\mathrm{CW}$ is 15.17 mas, and our estimates from the 1962-1977 series are about 11.3 and 14.6 mas for $x$ - and $y$-components, respectively. Obviously, our synthetic result is consistent with our observed results. If we only consider this fact, we may conclude that the observed target wobble from the 1962-1977 series very likely originates from the frequency modulation of $\mathrm{CW}$. But, here we do not want to infer whether the $\mathrm{CW}$ is frequency modulated, but obviously, the synthetic results for the 1978-1994 and 1995-2013 series based, respectively, on $M=0.23$ and 0.38 clearly show the appearance of the target wobble, whereas there is no significant peak for the target wobble in the corresponding actually observed spectra. Namely, the modulation index $M=0.23$ or 0.38 of $\mathrm{CW}$ cannot explain the observed results from the later two subseries. Although we cannot ensure that $M$ is a constant, we try to find an $M$ that can produce sufficient modulation for all the three sub-series. We find that $M=0.5$ is a good choice.

Now we set $M=0.5$, and construct three synthetic noisefree time series based on Eq. (2) for the 1962-1977, 1978- 

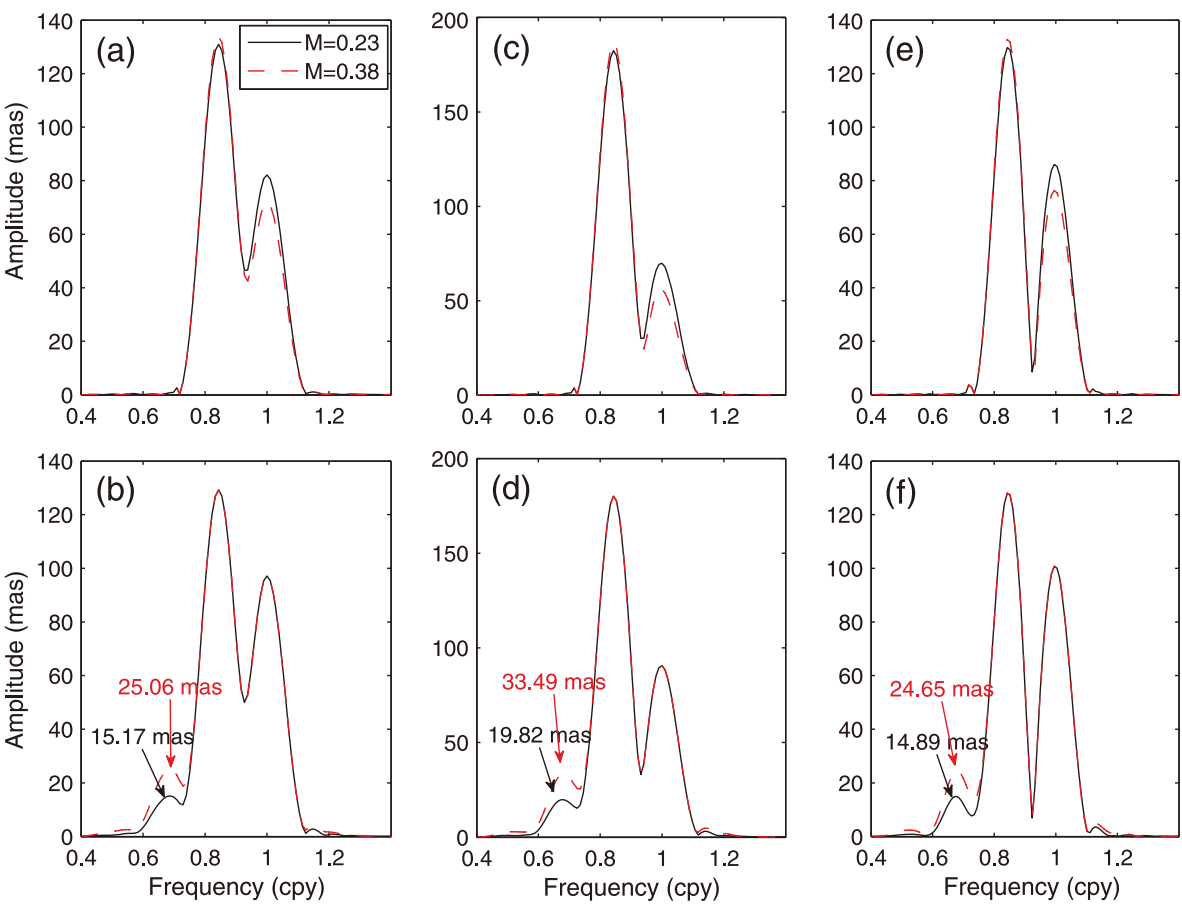

Figure 6. The amplitude spectra of the synthetic records (noise-free) based on a conventional approach (a-f) for the $x$-component of the 1962-1977 series, 1978-1994 series, and 1995-2013 series, respectively. The three top figures show the results without considering the frequency modulation of $\mathrm{CW}$, whereas the three bottom figures are the corresponding results considering the frequency modulation of $\mathrm{CW}$, with the modulation index $M=0.23$ (black curves) and 0.38 (red dashed curves).

Table 4. The input parameters for the six synthetic series. (FM: frequency modulation.)

\begin{tabular}{llrrrrrr}
\hline & & \multicolumn{5}{c}{ Synthetic series } \\
& & I & II (FM) & III & IV (FM) & V & VI (FM) \\
\hline \multirow{2}{*}{ CW } & Frequency (cpy) & 0.8437 & 0.8437 & 0.8437 & 0.8437 & 0.8437 & 0.8437 \\
& Amplitude (mas) & 137.6 & 137.6 & 191.3 & 191.3 & 136.4 & 136.4 \\
\hline \multirow{2}{*}{ AW } & Frequency (cpy) & 1.0000 & 1.0000 & 1.0000 & 1.0000 & 1.0000 & 1.0000 \\
& Amplitude (mas) & 63.7 & 63.7 & 42.6 & 42.6 & 67.4 & 67.4 \\
\hline \multirow{2}{*}{$531 \mathrm{dW}$} & Frequency (cpy) & 0.6875 & 0.6875 & 0.6875 & 0.6875 & 0.6875 & 0.6875 \\
& Amplitude (mas) & 44.1 & 44.1 & 50.0 & 50.0 & 31.2 & 31.2 \\
\hline
\end{tabular}

1994, and 1995-2013 series, and still set different amplitudes for the $\mathrm{CW}$ and $\mathrm{AW}$ to guarantee that the theoretical spectra coincide with the observational spectra (the input parameters are the same as the parameters for the three series without considering the frequency modulation of $\mathrm{CW}$, which are listed in Table 4; note here that only CW and AW are considered). The corresponding results are shown in Fig. 7 (using the $x$-components as examples), and the amplitudes of the first sidebands caused by the frequency modulation of $\mathrm{CW}$ are marked by the arrows. The synthesis results ( $x$-components) show that the amplitudes of the $531 \mathrm{dW}$ in the three sub-series are, respectively, 33.36, 44.98, and 32.98 mas, while the corresponding results of the $x$ - and $y$ components in IMF 6 are 33.2 (for $x$ ) and 32.7 mas (for $y$ ),
45.3 (for $x$ ) and 44.1 mas (for $y$ ), and 33.3 mas (for $x$ or $y$ ). Clearly, when $M=0.5$, the results from the frequency modulation of $\mathrm{CW}$ are consistent with the corresponding results in IMF6. If the $531 \mathrm{dW}$ in IMF6 is really caused by the frequency modulation of $\mathrm{CW}$ with a modulation index $M=0.5$, the $531 \mathrm{dW}$ in IMF5 may be excited by some geophysical processes, such as the atmospheric-oceanic excitation (Wahr, 1983; Chen et al., 2010); then, we can appropriately explain the observations after using EEMD and the disappearance of the $531 \mathrm{dW}$ in the recent PM series. Here, we would like to further demonstrate that EEMD can directly demodulate a frequency-modulated time series.

We generate six synthetic noise-free time series. The length of the synthetic series I and II is equal to that of the 

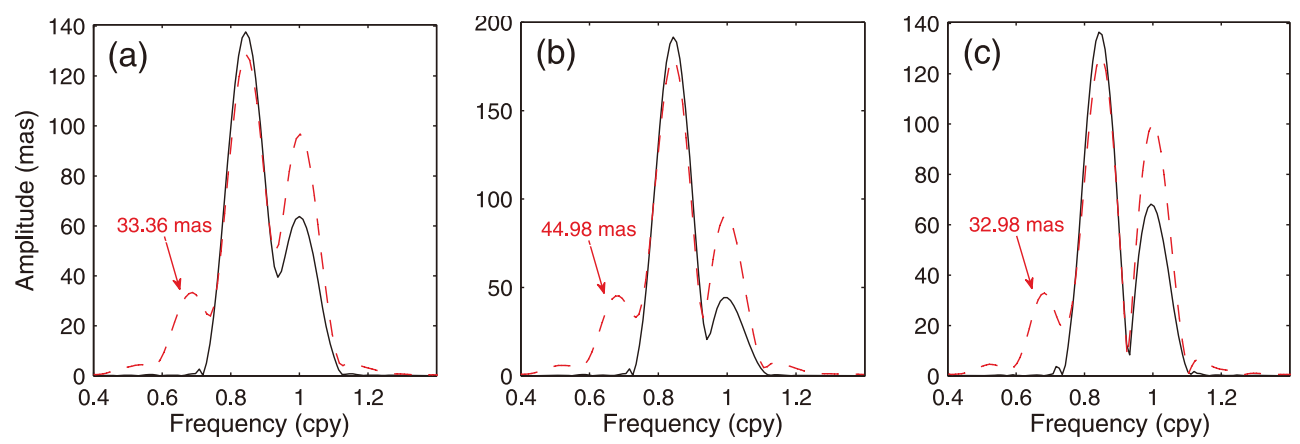

Figure 7. The amplitude spectra of the synthetic series without using EEMD. (a), (b), and (c) for the $x$-component of the 1962-1977, 1978-1994, and 1995-2013 series, respectively. The black curves indicate the results without considering the frequency modulation of CW, whereas the red dashed curves are the corresponding results when considering the frequency modulation of $\mathrm{CW}$, with the modulation index $M=0.5$.

1962-1977 series, the length of the synthetic series III and IV is equal to that of the 1978-1994 series, and the length of the synthetic series V and VI is equal to that of the 19952013 series, with a 1-day sampling interval. The synthetic series I (III/V) contains three sinusoidal components without frequency modulation, and the frequencies and amplitudes of $\mathrm{CW}$ and $\mathrm{AW}$ (see Table 4) are set to make the results for $\mathrm{CW}$ and $\mathrm{AW}$ after tuning the frequency modulation of $\mathrm{CW}$ to be the same as with the observations from the $x$-component of the 1962-1977 series (1978-1994 and 1995-2013; see Table 1 and Fig. $8 \mathrm{~b}-\mathrm{f}$ ). The third signal is the $531 \mathrm{dW}$, which is listed in Table 3. As for the synthetic series II (IV/VI), its parameters are the same as the synthetic series I (III/V), but a mechanism of the frequency modulation of $\mathrm{CW}$ is considered with the modulation index $M=0.5$. The spectra of the six synthetic series are shown in Fig. 8. Obviously, after considering the frequency modulation of $\mathrm{CW}$, the amplitude of the $531 \mathrm{dW}$ in the synthetic series II is reduced to only 11.05 mas, which is almost equal to the observed result obtained from the $x$-component of the 1962-1977 series without using EEMD (11.3 mas, see Fig. 3 and Table 1). The corresponding spectra of the IMF5 and IMF6 of the synthetic series II obtained after using EEMD are shown in Fig. 8b1 and b3. Similar to the real data, the phases for CW (or AW) in IMF5 and IMF6 are the same, whereas the phases for the $531 \mathrm{dW}$ in IMF5 and IMF6 are opposite to each other (Fig. 8b2). Comparing those synthetic results with the results from the $x$-component of the 1962-1977 series as listed in Table 1, the amplitudes of the $531 \mathrm{dW}$ in IMF5 (44.1 mas) and IMF6 (33.2 mas) of the real data are almost equal to those of the synthetic results. The results as shown in Fig. 8c-d and e-f have a similar nature to the results as shown in Fig. 8a-b. Namely, the input $531 \mathrm{dW}$ signal is in fact demodulated into IMF5, whereas the $531 \mathrm{dW}$ signal caused by the frequency modulation of CW is demodulated into IMF6.

The frequency modulation mechanism of $\mathrm{CW}$ is an open question. If we accept the modulation mechanism of $\mathrm{CW}$ addressed above, the results obtained from the PM series af- ter using EEMD can be appropriately explained. However, the excitation sources of the $531 \mathrm{dW}$ in IMF5 still cannot be confirmed. We do not intend to answer this question in this study, but we will try to confirm the $531 \mathrm{dW}$ in the independent gravity records in the following section to further confirm its presence.

\subsection{Results from the SG records}

If the $531 \mathrm{dW}$ can be excited by the atmospheric/oceanic angular momentum, or if it is the normal mode of the Earth, it may be found in the gravity records. Hence, we choose the superconducting gravimeter (SG) records from the Global Geodynamic Project (GGP) network to further confirm the existence of this signal. The GGP network has been operating about 25 years (from 1997), but the longest available continuous records (without very large gaps) are only about 16 years long, namely, the records from SG stations Membach (mb) (1995-2011) and Medicina (mc) (1998-2014). After removing the tidal effects (solid and ocean), the local atmospheric effect (without the $531 \mathrm{dW}$ signal in it), and the pole tides, the spectra of the residual gravity records are shown in Fig. 9 (see the black curves). Two peaks around $531 \mathrm{dW}$ can be found in Fig. 9a (for station mb) and b (for station mc). We have confirmed that the local atmospheric effect and the pole tides have no contributions to the $531 \mathrm{dW}$. Since the pole tides have been removed, namely, CW, AW and the possible $531 \mathrm{dW}$ signals caused by the polar motion have been removed from the gravity records, the residual $531 \mathrm{dW}$ must be caused by some other sources. Here, we further consider the hydrological loading (global) effect except the atmospheric loading (global) and non-tidal ocean loading effects (Petrov and Boy, 2004). The two latter effects are the same possible sources that excite the $531 \mathrm{dW}$ in PM series. However, Fig. 9 clearly shows that those three effects cannot explain the residual $531 \mathrm{dW}$; only the residual AW can be appropriately explained. This finding seems to be inconsistent with previous studies, but they can be explained. As Wahr (1983) 

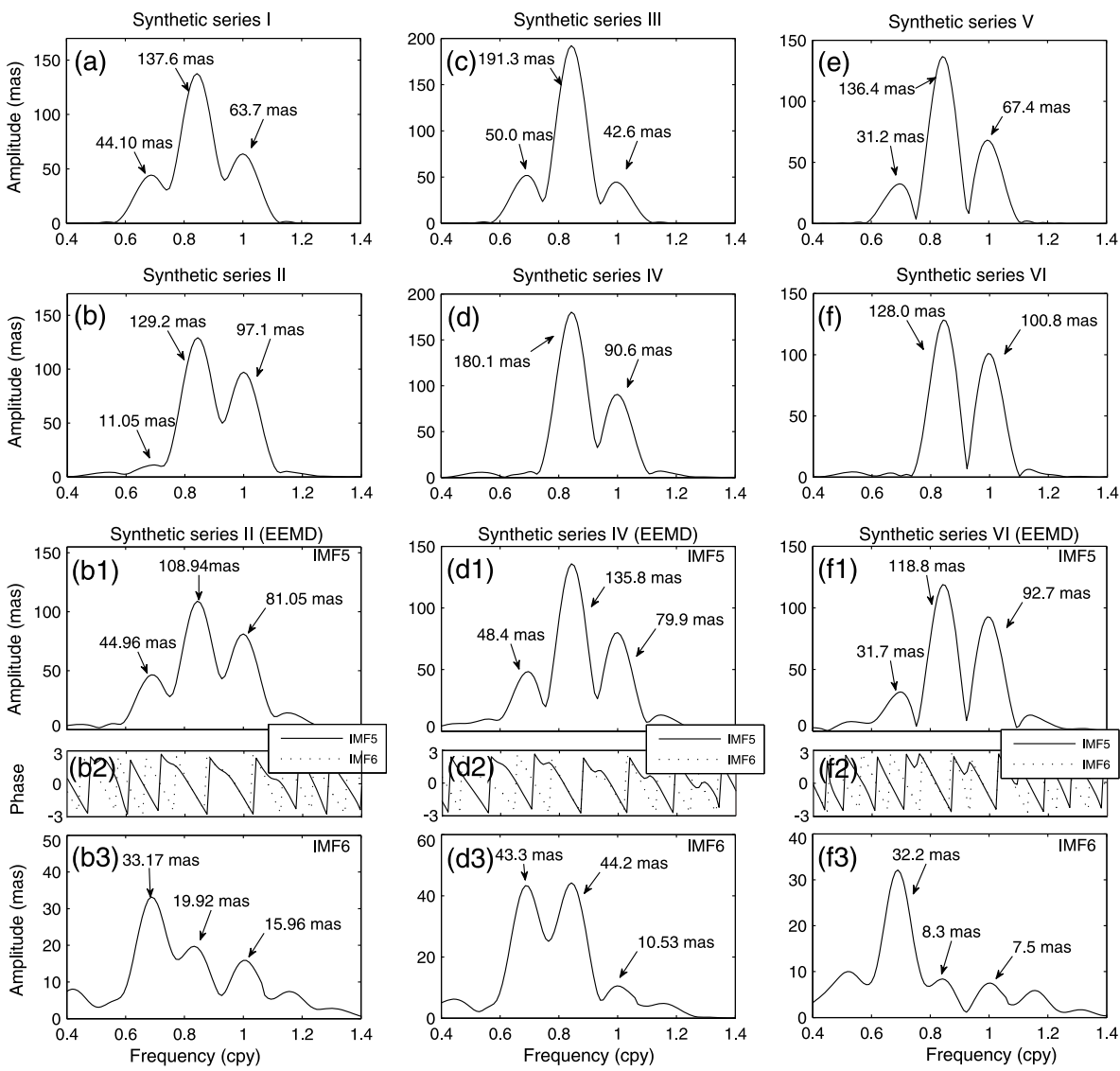

Figure 8. The amplitudes of the significant peaks (from left to right: $531 \mathrm{dW}, \mathrm{CW}, \mathrm{AW}$ ) are marked by the arrows. The spectra of synthetic series I (a) and II (b); and the spectra of the IMF5 and IMF6 of synthetic series II after using EEMD (b1-b3). The spectra of synthetic series III (c) and IV (d); and the spectra of IMF5 and IMF6 of synthetic series IV after using EEMD (d1-d3). The spectra of synthetic series V (e) and VI (f); and the spectra of the IMF5 and IMF6 of synthetic series VI after using EEMD (f1-f3).

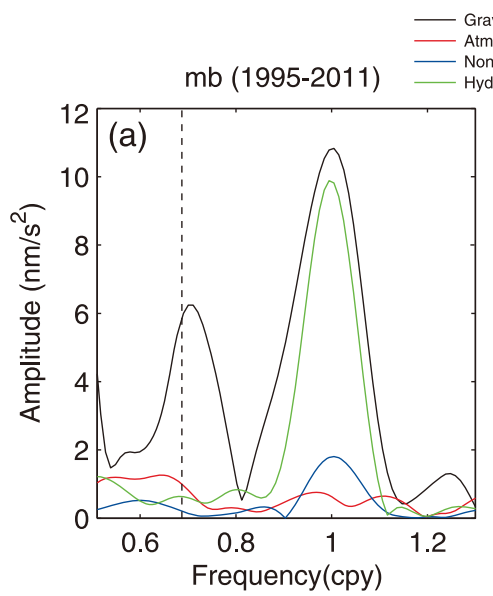

mc (1998-2014)

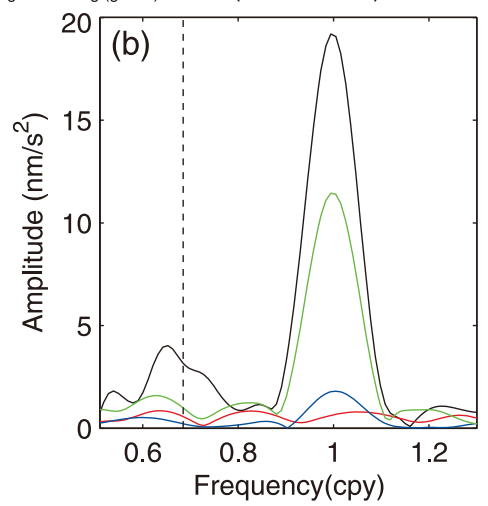

Figure 9. The Fourier spectra of the two residual SG records. (a) mb record; (b) mc record. The effects of the atmospheric loading (global), non-tidal ocean loading and the hydrological loading (global) are indicated by the red, blue and green curves, respectively.

and Chen et al. (2010) suggested, the $531 \mathrm{dW}$ can be excited by atmospheric/oceanic effects, and the excited signal by atmospheric or oceanic effects can be found in the PM se- ries only after convoluting with the $\mathrm{CW}$ term. However, the $531 \mathrm{dW}$ signals excited by atmospheric and oceanic effects seem to have different phases; hence, the $531 \mathrm{dW}$ cannot be 
found in the PM series (Chen et al., 2010). If we accept this, the $531 \mathrm{dW}$ signal in gravity records comes from two different sources: indirectly from polar motion that is affected by the atmospheric/oceanic effects, and directly from the atmospheric/oceanic effects. The excited $531 \mathrm{dW}$ from PM has been removed by taking away the pole tide effects. The excited $531 \mathrm{dW}$ directly from the atmospheric/oceanic effects has just the same nature as the excitation in the PM; if no convolution is processed with the $\mathrm{CW}$, the $531 \mathrm{dW}$ signal cannot be found in the PM or gravity records. Hence, our findings are actually consistent with previous studies.

No matter how, our results clearly show that the demodulation feature of EEMD is helpful for detecting the $531 \mathrm{dW}$ signal in the PM series, and we confirm that the amplitude and frequency of the $531 \mathrm{dW}$ vary with time.

\section{Discussion and conclusion}

After applying EEMD to the 1962-2013 PM time series (EOP C04), a $531 \mathrm{dW}$ is clearly found with a mean amplitude of about 7 mas (with much larger amplitudes in the IMF5 and IMF6 series, respectively), but in the spectra without using EEMD, this signal cannot be found. The $531 \mathrm{dW}$ has been found by previous studies in the 1962-1977 PM series with a lower SNR. To confirm previous observations (Carter, 1981, 1982) and to further study this signal, we divide the whole PM series into three sub-series, the 1962-1977, 1978-1994 and 1995-2013 PM time series. Without using EEMD, the results for the 1962-1977 PM series are consistent with previous studies, while the $531 \mathrm{dW}$ signal disappears from the Fourier spectra of the 1978-1994 and 1995-2013 PM time series. However, after applying EEMD to those three subseries, the $531 \mathrm{dW}$ signals can be found in each sub-series with different outstanding amplitudes (based on the spectra of the decomposed IMF5 and IMF6 series), which (taking $x$-components as examples) are, respectively, about 12.8 in 1962-1977, 4.0 in 1978-1994, and 3.4 mas in 1995-2013 (see Table 2), while the corresponding noise levels of the three sub-series are about 9.5, 8.8, and 5.4 mas, respectively. That is the reason why the $531 \mathrm{dW}$ signal can only be directly found in the 1962-1977 PM series without using EEMD.

Although the frequency modulation mechanism of $\mathrm{CW}$ is an open question, we find that if the modulation index $M$ of $\mathrm{CW}$ equals 0.5 , the results obtained from the PM series after using EEMD can be appropriately explained. Furthermore, using synthetic tests, we confirmed the demodulation feature of EEMD, which can help us find the $531 \mathrm{dW}$ signal in the PM series, but this paper does not attempt to explain the possible excitation sources of the $531 \mathrm{dW}$. Given that the $531 \mathrm{dW}$ signal can be directly found in the SG records without using EEMD, it should be considered in the study of the long-period effects in some relevant geophysical data sets.

Though various previous studies have confirmed that the incomplete orthogonality of EEMD hardly affects the anal- ysis of many geophysical processes, it might still affect our PM time series analysis process. Owing to the frequency and amplitude of the $531 \mathrm{dW}$ signal being time-variant (generally, they might be caused by the excited process and the background noise in different time spanning), it becomes quite difficult to explore its excitations. It might be caused by the core dynamics, or even by some random processes (e.g., Chao et al., 2014), but from the phase results of the $531 \mathrm{dW}$ of King (2015), we may prefer to consider that the $531 \mathrm{dW}$ seems to be excited by some kind of unknown process, which needs further investigations. Anyway, as a final conclusion, we suggest that the $531 \mathrm{dW}$ signal exists in nature, but left its mechanism open. With the accumulation of high-precision geodetic observation data, the long-period terms, such as $531 \mathrm{dW}$, are to be further studied, and this may help us better understand the Earth system.

Acknowledgements. We thank Jim Ray, Wei Chen and Benjamin Fong Chao for fruitful discussions that improved the manuscript, and thank Christian Bizouard for helpful comments on an early draft of this paper. We also thank M. King and two anonymous reviewers for their valuable comments and suggestions, which significantly improved the manuscript. The atmospheric/hydrological/non-tidal ocean loadings are provided by J. P. Boy (which can be downloaded from http://loading.u-strasbg.fr/GGP/index.html). This study is supported by NSFC (grant no. 41174011), National 973 Project China (grant no. 2013CB733305), and NSFC (grant nos. 41210006 and 41429401).

Edited by: R. Gloaguen

Reviewed by: M. King and two anonymous referees

\section{References}

Carter, W. E.: Frequency modulation of the Chandlerian component of polar motion, J. Geophys. Res., 86, 1653-1658, 1981.

Carter, W. E.: Refinements of the polar motion frequency modulation hypothesis, J. Geophys. Res., 87, 7025-7028, 1982.

Chambers, D. P.: Evaluation of empirical mode decomposition for quantifying multi-decadal variations and acceleration in sea level records, Nonlin. Processes Geophys., 22, 157-166, 2015, http://www.nonlin-processes-geophys.net/22/157/2015/.

Chao, B. F.: Autoregressive harmonic analysis of the Earth's polar motion using homogeneous International Latitude Service data, J. Geophys. Res., 88, 10299-10307, 1983.

Chao, B. F. and Gilbert, F.: Autoregressive estimation of complex eigenfrequencies in low frequency seismic spectra, Geophys. J. Roy. Astron. Soc., 63, 641-657, 1980.

Chao, B. F., Chung, W. Y., Shih, Z. R., and Hsieh, Y. K.: Earth's rotation variations: a wavelet analysis, Terra Nova, 26, 260-264, 2014.

Chen, W., Shen, W. B., Han, J., and Li, J.: Free wobble of the triaxial Earth: theory and comparisons with International Earth Rotation Service (IERS) data, Surv. Geophys., 30, 39-49, 2009. 
Chen, W., Shen, W. B., and Dong, X. W.: Atmospheric Excitation of Polar Motion, P. Soc. Photo-Opt. Ins., 13, 130-136, 2010.

Chen, W., Ray, J., Li, J., Huang, C., and Shen, S.: Polar motion excitations for an Earth model with frequency-dependent responses: 1. A refined theory with insight into the Earth's rheology and core-mantle coupling, J. Geophys. Res., 118, 1-20, 2013a.

Chen, W., Ray, J., Li, J., Shen, S., and Huang, C.: Polarmotion excitations for an Earth model with frequency-dependent responses: 2. Numerical tests of the meteorological excitations, J. Geophys. Res., 118, 1-13, 2013b.

Ding, H. and Chao, B. F.: Detecting harmonic signals in a noisy time-series: the $z$-domain Autoregressive (AR-z) spectrum, Geophys. J. Int., 201, 1287-1296, 2015.

Ding, H. and Shen, W. B.: Determination of the complex frequencies for the normal modes below $1 \mathrm{mHz}$ after the 2010 Maule and 2011 Tohoku earthquakes, Ann. Geophys., 56, 5, doi:10.4401/ag6400, 2013.

Franzke, C.: Multi-scale analysis of teleconnection indices: climate noise and nonlinear trend analysis, Nonlin. Processes Geophys., 16, 65-76, doi:10.5194/npg-16-65-2009, 2009.

Gross, R. S., Fukumori, I., and Menemenlis, D.: Atmospheric and oceanic excitation of the Earth's wobbles during 1980-2000, J. Geophys. Res., 108, 2370, doi:10.1029/2002JB002143, 2003.

Höpfner, J.: Chandler and annual wobbles based on space-geodetic measurements, J. Geodyn., 36, 369-381, 2003.

Huang, N. E., Shen, Z., Long, S. R., Wu, M. C., Shih, H. H., Zheng, Q., Yen, N. C., Tung, C. C., and Liu, H. H.: The empirical mode decomposition and the Hilbert spectrum for nonlinear and nonstationary time series analysis, Proc. Roy. Soc. Lond., A454, 903-995, 1998.

Huang, N. E., Shen, Z., Long, S. R., Wu, M. C., Shih, H. H., Zheng, Q., Yen, N. C., Tung, C. C., and Liu, H. H.: A review on Hilbert-Huang transform: Method and its applications to geophysical studies, Rev. Geophys., 46, RG2006, doi:10.1029/2007RG000228, 2008.

Jackson, L. P. and Mound, J. E.: Geomagnetic variation on decadal time scales: What can we learn from Empirical Mode Decomposition?, Geophy. Res. Lett., 37, L14307, doi:10.1029/2010GL043455, 2010.

King, M.: Interactive comment on "Search for the 531 day-period wobble signal in the polar motion based on EEMD" by H. Ding and W. B. Shen, Nonlin. Processes Geophys. Discuss., 2, C163C168, 2015.

King, M. A. and Watson, C. S.: Geodetic vertical velocities affected by recent rapid changes in polar motion, Geophys. J. Int., 199, 1161-1165, 2014.
Lee, T., and Ouarda, T. B. M. J.: Prediction of climate nonstationary oscillation processes with empirical mode decomposition, J. Geophys. Res., 116, D06107, doi:10.1029/2010JD015142, 2011.

Liu, H. Y., Lin, Z. S., Qi, X. Z., Li, Y. X., Yu, M. T., Yang, H., and Shen, J.: Possible link between Holocene East Asian monsoon and solar activity obtained from the EMD method, Nonlin. Processes Geophys., 19, 421-430, doi:10.5194/npg-19-4212012, 2012.

Morgan, P. J., King, R. W. and Shapiro, I. I.: Spectral analysis of variation of latitude derived from lunar laser ranging and satellite Doppler observations (abstract), Eos Trans., AGU, 63, p. 302, 1982.

Na, S., Cho, J., Baek, J., Kwak, Y., Yoo, S., Cho, S., Lim, H., Kwak, Y., Park, J., and Park, P.: 500-day period component in the Earth's polar motion (abstract). AGU Fall Meeting, San Francisco, California, USA, 2011/11, G53B-0910, 2011.

Okubo, S.: Is the Chandler period variable? Geophys. J. R. Astron. Soc., 71, 629-646, 1982.

Pan, C.: Linearization of the Liouville equation multiple splits of the Chandler frequency Markowitz wobbles and error analysis. International J. Geosci., 3, 930-951, 2012.

Pee, M. C. and McMahon, T. A.: Recent frequency component changes in interannual climate variability, Geophys. Res. Lett., 33, L16810, doi:10.1029/2006GL025670, 2006.

Petrov, L. and Boy, J.-P.: Study of the atmospheric pressure loading signal in VLBI observations, J. Geophys. Res., 109, B03405, doi:10.1029/2003JB002500, 2004.

Seitz, F. and Schmidt, M.: Atmospheric and oceanic contributions to Chandler wobble excitation determined by wavelet filtering, J. Geophys. Res., 110, B11406, doi:10.1029/2005JB003826, 2005.

Shen, W. B. and Ding, H.: Observation of spheroidal normal mode multiplets below $1 \mathrm{mHz}$ using ensemble empirical mode decomposition, Geophys. J. Int., 196, 1631-1642, 2014.

Tary, J. B., Herrera, R. H., Han, J., and van der Baan, M.: Spectral estimation - What is new? What is next?, Rev. Geophys., 52, 723-749, 2014.

Thomas, E. R., Dennis, P. F., Bracegirdle, T. J., and Franzke, C.: Ice core evidence for significant 100-year regional warming on the Antarctic Peninsula, Geophys. Res. Lett., 36, L20704, doi:10.1029/2009GL040104, 2009.

Vicente, R. O. and Wilson, C. R.: On the variability of the Chandler frequency, J. Geophys. Res., 102, 20439-20446, 1997.

Wahr, J.: The effects of the atmosphere and oceans on the Earth's wobble and on the seasonal variations in the length of day, II. Results, Geophys. J. R. Astr. Soc., 74, 451-487, 1983.

Wu, Z. H. and Huang, N. E.: Ensemble empirical mode decomposition: a noise-assisted data analysis method, Adv. Adapt. Data. Anal., 1, 1-41, 2009. 